\title{
Preoperative Concurrent Chemoradiotherapy with Oral Fluo- ropyrimidine in Locally Advanced Rectal Cancer: How Good Is Good Enough?
}

\author{
Hyun Cheol Chung, M.D., Ph.D. \\ Yonsei Cancer Center, Cancer Metastasis Research Center, Yonsei University College of Medicine
}

In this issue of the Cancer Treatment and Research, Park et al (1) presented a prospective study on the efficacy and toxicity of combined chemoradiotherapy delivered preoperatively for locally advanced rectal cancer. The role of concurrent chemoradiotherapy in stage II and III rectal cancer has been established by postoperative chemoradiation treatment. While efforts to compare the efficacy and toxicity of preoperative versus postoperative concurrent chemoradiation treatment in phase III trials in the United States have been largely unsuccessful, due to a poor patient accrual rate, a German trial has shown a benefit in pelvic control with preoperative chemoradiation compared to postoperative chemoradiation (2). Furthermore, the Swedish Rectal Trial Group have shown benefits in both pelvic control and overall survival with preoperative radiotherapy alone. A randomized trial has not yet been completed comparing the role of preoperative chemoradiotherapy to radiation alone. However, the rationale for preoperative concurrent chemoradiotherapy is based on extrapolation from postoperative trials. The advantages of preoperative over postoperative treatment include; less acute toxicity and an increased sphincter preservation rate. The most significant disadvantages of preoperative treatment are that a certain fraction of patients will be treated unnecessarily due to the inaccuracy of clinical staging, which will result in treatment failure due to inherited treatment resistance.

The authors addressed the very relevant question of whether protracted venous infusion (PVI) of 5-fluorouracil (5-FU) with concurrent radiotherapy could be replaced by capecitabine. The concurrent capecitabine and radiotherapy was well tolerated in a phase I tria, with minimal toxicity (3). Over the past decades, evidence has been accumulated on the effectiveness and limitation of 5-fluorouracil with biochemical modulation. Weekly infusional 5-fluorouracil is less toxic and equally active as Mayo regimen. Biweekly infusional 5-flurouracil with leucovorin has also been shown to be less toxic and more active than the Mayo schedule. Capecitabine is less toxic than the Mayo regimen, with an as good or better response. Irinotecan

Correspondence: Hyun Cheol Chung, Yonsei Cancer Center, Cancer Metastasis Research Center, Yonsei University College of Medicine, 134 Sinchon-dong, Seodaemun-gu, Seoul 120-752, Korea. (Tel) 82-2-361-7652, (Fax) 82-2-393-3652, (E-mail) unchung8@ yumc.yonsei.ac.kr plus a weekly bolus of 5-fluorouracil plus leucovorin has an improved activity over that of the Mayo regimen. However, attempts to add irinotecan or oxaliplatin to the Mayo regimen resulted in unacceptable toxicity, with no improved benefit. The authors treated the patients with oral fluoropyrimidines, given concurrently with radiotherapy. The primary end point of the study was the pathologic response of the primary lesion to the preoperative treatment. This was well-designed and conducted, with results showing low acute toxicity as well as good pathologic tumor response and local tumor control, with sphincter preservation and treatment compliance comparable with the results from other studies evaluating preoperative chemoradiotherapy. On this base, capecitabine, which is approved for metastatic colorectal cancer, is a good candidate for a phase III study in combination with radiotherapy. The National Surgical Adjuvant Breast and Bowel Project (NSABP) R-04 protocol was designed as a phase III trial for the comparison of concurrent oral fluoropyrimidine with PVI of 5-FU in locally advanced rectal cancer. This protocol initially incorporated UFT, but later capecitabine replaced UFT in this trial, following its approval, therefore, UFT was abandon in the United States. The NSABP R-04 protocol has been on hold for 2 years pending final approval by the National Cancer Institute.

Patients who do not have direct involvement of the levator or sphincter muscles are candidate for sphincter preservation. Preoperative chemoradiation can reduce the tumor size and thus allows transection of the rectum at the anal canal with an adequate margin. The questions are: "What is the impact of preoperative chemoradiotherapy on the downstaging, sphincter preservation, and subsequent recurrence and survival". Twentyseven years after the first accrual of patients to the GITSG 7175 protocol, many new therapeutic issues still remain. It is important to recognize the difference between downsizing and downstaging. The authors now raise an issue on the accurate evaluation of the efficacy and safety of the sphincter preservation rate by downsizing rectal cancer with preoperative chemoradiation. The authors report they were able to perform sphincter saving operations on most patients $(73 \%)$ in the study, with a $74 \%$ overall downstaging rate. From a surgical viewpoint, high-risk rectal cancers are downsized, even when they are not downstaged.

After preoperative chemoradiation, the clinical tumor response is notoriously unreliable as a predictor of the pathological response. This may render patient selection for sphincter preservation secondary to that of downsizing based on the 
clinical evaluation. In the Lyon R0-04 trial, the objective clinical response rate was $75 \%$, with $45 \%$ of these patients showing pathological CR or a microscopic residual disease (4). In a phase II concurrent trial, with an oral agent (tegafur-uracil modulated with leucovorin), the clinical response and histologically proven downstaging rate were 63 and $60 \%$, respectively, with reasonable toxicity and postoperative complications (5). The only independent significant prognostic factor for pelvic recurrence is a clinical $\mathrm{T}$ stage confirmed by involvement of an adjacent organ on physical examination, CT or endoscopic ultrasound. A response to chemoradiotherapy (microscopic or no residual disease) is suggested as a positive predictor of an improved overall survival $(p=0.07)(6)$. The majority of patients who show a complete response in the primary tumor will have residual cancer within the nodes and operative specimens (7). The authors addressed this point in assessing the pathological $\mathrm{T}$ and $\mathrm{N}$ stages (T-factor downstaging in 59\% and $\mathrm{N}$-factor downstaging in $51 \%$ ). The fact is, $90 \%$ of the patients studied in this report had viable residual cancer cells in their resected specimens. Based on these kinds of findings, the $\mathrm{T}$ downstage without nodal involvement was recently suggested as a positive predictor of disease free survival (8).

Total mesorectal excision (TME) emphasizes the need for complete dissection of the mesorectum with the lymph node bearing portion of the rectum. TME seems to be superior in sphincter preservation, local tumor control and survival compared to traditional rectal surgery (9). Hence, better quality TME could result in a lower local recurrence rate, with the subsequent lesser benefit of preoperative chemoradiotherapy. In support of this hypothesis, the Swedish Rectal Cancer Trial compared traditional surgery both with and without short course preoperative radiotherapy. The local recurrence rates in the radiated and non-radiated patients were 11 and $27 \%$, respectively. The rate of pelvic recurrence after 5-FU-based chemoradiotherapy, followed by surgery, has been reported between 2 and $10 \%$ in a single arm study, and $2.4 \%$ with radiation alone, followed by surgery, in a phase III trial (10). In an effort to further improve the results of preoperative chemoradiation, clinicians are experimenting with increasing the radiation dosage using a concomitant booster technique (11).

All of the promising novel agents approved for colorectal cancer (irinotecan, oxaliplatin, cetuximab, bevacizumab) have radiosensitizing activity, and some have already reported preliminary results of increased local tumor control (12). Because twice as many patients ultimately develop distant metastasis compared with local recurrence after surgery, new trials with novel systemic chemotherapy, in addition to radiosensitization, will be more appealing to the clinicians. For this reason, direct comparison of capecitabine with intravenous 5-FU will be difficult in the future, due to a limited patient pool and budget. In this situation, level I evidence must be obtained that can support substituting oral fluoropyrimidines for intravenous 5-FU with radiotherapy. The two adjuvant colon cancer trials (Xeloda Adjuvant Colon Cancer trial [X-ACT] and NSABP C-06) addressing the role of oral fluoropyrimidines are showing promise. X-ACT trials have favored capecitabine over bolus 5-FU and leucovorin (Mayo regimen) in relation to the toxicity. If these trials support that oral fluoropyrimidines are equivalent or superior to 5-FU plus leucovorin, then con- sidering the INT-0144 trial, which have shown identical survival between 5-FU plus leucovorin and levamisol plus radiotherapy and that of PVI of 5-FU plus radiotherapy, these data will support the substitution of oral fluoropyrimidines for PVI of 5-FU with radiotherapy.

In summary, a phase II trial evaluating capecitabine with radiotherapy, presented by $\mathrm{Kim}$, et al. in this issue, demonstrated a low local recurrence and high sphincter preservation, with minimal acute toxicity. The NSABP R-04 trial will support the rationale of this substitution. The use of salvage therapy with irinotecan or oxaliplatin will be investigated in non-responders to this regimen.

\section{REFERENCES}

1. Park J, Kim JH, Ahn SD, Lee S, Shin SS, Kim JC, et al. Prospective phase II study of preoperative chemoradiation with capecitabine in locally advanced rectal cancer. Cancer Res Treat. 2004;36:354-9.

2. Sauer R. German Rectal Cancer Group. Adjuvant versus neoadjuvant combined modality treatment for locally advanced rectal cancer. First result of the German Rectal Cancer Study (CAO/ARO/AIO-94). Int J Radiat Oncol Biol Phys. 2003;57: S124-5(supple 2).

3. Dunst J, Reese T, Sutter T, Zuhlke H, Hinke A, KollingSchlebusch K, et al. Phase I trial evaluating the concurrent combination of radiotherapy and capecitabine in rectal cancer. J Clin Oncol. 2002;20:3983-91.

4. Gerard JP, Chapet O, Nemoz C, Romestaing P, Mornex F, Coquard R, et al. Preoperative concurrent chemoradiotherapy in locally advanced rectal cancer with high-dose radiation and oxaliplatin-containing regimen: The Lyon R0-04 phase II trial. J Clin Oncol. 2003;21:1119-24.

5. Uzcudun AE, Batlle JF, Velasco JC, Sanchez Santos ME, Carpeno Jde C, Grande AG, et al. Efficacy of preoperative radiation therapy for respectable rectal adenocarcinoma when combined with oral tegafur-uracil modulated with leucovorin: result from a phase II study. Dis Colon Rectum. 2002;45:1349-58.

6. Rich TA, Skibber JM, Ajani JA, Buchholz DJ, Cleary KR, Dubrow RA, et al. Preoperative infusional chemoradiation therapy for stage T3 rectal cancer. Int J Radiat Oncol Biol Phys. 1995;32:1025-9.

7. Meterissian S, Skibber J, Rich T, Roubein L, Ajani J, Cleary $\mathrm{K}$, et al. Patterns of residual disease after preoperative chemoradiation in ultrasound T3 rectal carcinoma. Ann Surg Oncol. 1994;1:111-6.

8. Janjan NA, Abbruzzese J, Pazdur R, Khoo VS, Cleary K, Dubrow $\mathrm{R}$, et al. Prognostic implications of response to preoperative infusional chemoradiation in locally advanced rectal cancer. Radiother Oncol. 1999;51:153-60.

9. MacFarlane JK, Ryall R, Heald RJ. Mesorectal excision for rectal cancer. Lancet. 1993;341:457-60.

10. Kapiteijn E, Marijnen CA, Nagtegaal ID, Putter H, Steup WH, Wiggers $\mathrm{T}$, et al. Preoperative radiotherapy combined with total mesorectal excision for resectable rectal cancer. N Engl J Med. 2001;345:638-46.

11. Mohiuddin M, Regine WF, John WJ, Hagihara PF, McGrath PC, Kenady DE, et al. Preoperative chemoradiation in fixed distal rectal cancer: dose time factors for pathological complete response. Int J Radiat Oncol Biol Phys. 2000;46:883-8.

12. Crane $\mathrm{CH}$, Ellis L, Xiong $\mathrm{H}$. Preliminary results of a phase I study of rhuMab VEGF (bevacizumab) with concurrent radiotherapy and capecitabine. ASCO GI Symposium, San Francisco, CA, January 2004 (abstr 85). 